Research Paper

\title{
Distinct profile of cell-free DNA in malignant pleural effusion of non-small cell lung cancer and its impact on clinical genetic testing
}

\author{
Yongfeng Yu, Jie Qian, Lan Shen, Wenxiang Ji, Shun $\mathrm{Lu}^{\varpi}$ \\ Shanghai Lung Cancer Center, Shanghai Chest Hospital, Shanghai Jiao Tong University, Shanghai, China. \\ $\triangle$ Corresponding author: Shun Lu, Shanghai Lung Cancer Center, Shanghai Chest Hospital, Shanghai Jiao Tong University, 241 West Huaihai Road, Xuhui \\ District, Shanghai 200030, China. E-mail: shunlu@sjtu.edu.cn. \\ (c) The author(s). This is an open access article distributed under the terms of the Creative Commons Attribution License (https://creativecommons.org/licenses/by/4.0/). \\ See http://ivyspring.com/terms for full terms and conditions.
}

Received: 2020.08.26; Accepted: 2020.12.18; Published: 2021.01.30

\begin{abstract}
Cell-free DNA (cfDNA) in supernatant of pleural effusion from advanced NSCLC patients has been proved as surrogate sample detecting therapeutic targets as well as tumor mutation burden (TMB). As recently reported, cfDNA in pleural effusion supernatant is superior to plasma in TMB evaluation. It is reasonable to hypothesize that cfDNA profile in pleural effusion (PE) and plasma might be different. It remains to be elucidated why cfDNA in PE supernatant impacts on genetic analysis. Consequently, the approach dealing with cfDNA from PE supernatant might need to be different from that for plasma cfDNA in order to obtain accurate clinical genetic testing result.

Methods: Pleural effusion samples from 32 patients with stage IV lung adenocarcinoma were collected. Supernatant and sediment were processed separately to extract Cell-free DNA as well as sediment DNA (PE-S). cfDNA from pleural effusion was analyzed by Agilent 2100 bioanalyzer. Libraries were prepared by 1 ) direct use of the total cfDNA without fragmentation step (PE-FL) or 2) use of full-length cfDNA fragmented to 150-250bp (PE-F), 3) use of cfDNA fragments enriched to 167bp (PE-E167) as well as 4) use of cfDNA fragments larger than 500bp enriched (PE-E500). All samples were subjected to targeted next-generation sequencing (NGS) with a panel of 448 cancer-related genes as well as a panel of 10 NSCLC driver genes.

Results: cfDNA were successfully extracted from 30 MPE samples. cfDNA displayed distinct profile in supernatant of malignant pleural effusion from that of plasma cfDNA. No statistical difference in detection of hotspot variations between PE-E167 and PE-F by 448-gene or 10-gene panel. While TMB from PE-F samples was significantly higher than that from PE-E167 and PE-FL. Higher TMB from PE-F was resulted from cancer-unspecific variants with low allele frequency $(0.1 \%-1 \%)$ which were mainly introduced by long-fragment cfDNA. Similar genetic profile was observed between paired cfDNA of PE-FL and cfDNA of PE-E167.

Conclusion: Long-fragment cfDNA in the PE supernatant will introduce low abundant cancer unrelated variants which leads overestimation of TMB. Paired PE-FL and PE-E167 gave comparable outcomes. Direct use of the total cfDNA without fragmentation step (PE-FL) is recommended for library preparation of NGS testing in clinical practice to exclude interference from long fragments of the cfDNA.
\end{abstract}

Key words: lung cancer; pleural effusion; cell-free DNA; long fragment; genetic analysis

\section{Introduction}

To take advantage of targeted therapy and immune checkpoint inhibitors (ICIs), molecular profiling is pre-requested [1-3]. In clinical practice, it is not always available to obtain sufficient tumor tissue for molecular profiling, especially from patients with advanced tumors. Thus, liquid biopsy, as an approach more feasible to assess circulating tumor DNA (ctDNA) by using blood or other body fluid samples like sputum [4] and malignant pleural effusion [5], has been proved to guide targeted 
therapy as well as predict efficacy of immunotherapy by yielding trustable results of driver gene mutations as well as bTMB respectively [6].

Malignant pleural effusion (MPE), a common complication of lung cancer, defined as excessed fluid accumulated between lung and pleural cavity, is significantly associated with patients' poor prognosis. Drainage of excessive MPE provides a good opportunity to obtain sufficient source to assess tumor genomics [7]. Sedimental cell pellet has been used for cytological diagnosis as well as genetic testing [8]. While, due to the complexity of cell compositions, especially in hemorrhagic PEs [9], large numbers of erythrocytes as well as leukocytes, would largely impact the sensitivity of detecting somatic mutation. Cell-free DNA (cfDNA) in the supernatant of MPE has been proved to be superior to sedimental cell pelleting in detection of genetic variants as well as tumor mutation burden (TMB). Moreover, as Tong et al. recently showed, tumor-derived DNA from PE supernatant can be a better sample source than plasma cfDNA for assessing genetic aberrations as well as TMB in patients with advanced NSCLC when tumor tissue is not available [10]. In addition to higher DNA concentration in MPE giving rise to higher TMB evaluation, differential cfDNA fragment profile in MPE could be a putative factor making genetic testing different. While, by far, cfDNA profile in MPE was not thoroughly investigated.

Fragment size of cfDNA is heterogeneous in multiple types of body fluid [11]. In plasma, peak cfDNA fragments are approximately $167 \mathrm{bp}$, accompanied by sizes in multiples of $167 \mathrm{bp}$ due to apoptosis induced nucleosome releasing. While long-fragment cfDNA, with size of more than 10,000 $\mathrm{bp}$, was reported by previous study on plasma cfDNA samples [12]. Our preliminary data displayed that, compared with plasma, cfDNA in supernatant of MPE contains much more longer DNA fragments with size of longer than $500 \mathrm{bp}$ (Figure 1A, 1B). However, whether those longer cfDNA fragments in PE supernatant will have impact on genetic testing, and how to deal with these longer fragments in clinics is still unclear.

In this study, cfDNA in supernatant of MPE was extracted followed by comprehensive analysis of driver mutations as well as TMB by NGS applying 10-gene and 448-gene panel respectively. Fragmented full-length cfDNA (PE-F, n=30) in supernatant of MPE which contained entire genetic information was prepared. Further, the impact of PE-F on genetic analysis was thoroughly investigated by comparing tested results with PE-FL (direct library preparation from the total cfDNA without fragmentation step) or PF-E167 (enriched $167 \mathrm{bp}$ fragments). To clarify the influence of long-fragment cfDNA on detecting genetic aberrations, differences of genetic aberrations between long-fragment cfDNA and PE-E167 or PE-FL were investigated.

\section{Materials and Methods}

\section{Patient recruitment and sample collection}

From May 2018 to February 2019, 32 patients with stage IV, pathologically confirmed lung adenocarcinoma were enrolled. MPE were drained and collected due to relieving clinical symptoms at diagnosis or during disease progression at Shanghai Chest Hospital of Shanghai Jiao Tong University. Clinicopathological characteristics and survival data of 32 patients with lung cancer were obtained and combined with high-throughput sequencing data for joint analysis. Patients' baseline data are provided in Table 1. This study was approved by the ethics committee of Shanghai Chest Hospital of Shanghai Jiao Tong University, and all patients provided informed written consent. All samples were tested at a clinical genomics testing laboratory (AmoyDx, Xiamen, China) using protocols approved by the ethics committee of Shanghai Chest Hospital of Shanghai Jiao Tong University.

MPE were collected simultaneously from all 32 patients. Fifty milliliters of MPE was collected into a $50 \mathrm{ml}$ sterile centrifuge tube (Corning, NY, USA) and centrifuged at $3000 \mathrm{~g}$ for $10 \mathrm{~min}$ at room temperature to separate the PE supernatant and PE cell sediment, which were subsequently used for cfDNA and genomic DNA (gDNA) extraction, respectively. Then, 2-4 ml of anticoagulated whole blood was collected in EDTA anticoagulation tubes (BD, CAT, USA), and centrifuged at $1600 \mathrm{~g}$ for $10 \mathrm{~min}$ at room temperature to isolate white blood cells for gDNA extraction and as a germline control.

\section{DNA extraction, enrichment, and library construction}

CfDNA was extracted from PE supernatant using the QIAamp Circulating Nucleic Acid Kit (Qiagen, Hilden, Germany) following the standard protocol. gDNA from PE cell sediment and white blood cells were extracted using the AmoyDx Blood and Leukocyte DNA Kit (AmoyDx, Xiamen, China). All DNA was quantified using the QuantFluor ${ }^{\circledR}$ dsDNA System on a Quantus ${ }^{\mathrm{TM}}$ Fluorometer (Promega, USA). CfDNA from thirty samples were successfully extracted. Fragment distribution was analyzed in a Bioanalyzer 2100 using the High Sensitivity DNA Kit (Agilent Technologies, Santa Clara, CA). Full-length cfDNA from PE supernatant without fragmentation step during NGS library 
preparation was termed PE-full-length (PE-FL, Figure 1B) (11 of 30 samples). To obtain all information from cfDNA in PE supernatant, full length cfDNA was fragmented to a size range from $150 \mathrm{bp}$ to $250 \mathrm{bp}$ using Covaris M220 (Covaris, Woburn, MA) (terminated as PE-F, 30 samples, Figure 1C). CfDNA from PE supernatant was separated based on size using Agencourt Ampure XP beads (Beckman Coulter, Indianapolis, IN). AMPure XP beads in $0.6 \times$ and $1.2 \times$ and $0.6 \times$ volumes were used to enrich DNA $\sim 167$ bp (PE-Enrichment 167 [terminated as PE-E167, 23 of 30 samples], Figure 1D) and > $500 \mathrm{bp}$ (PE-enrichment 500 [PE-E500], 6 of 30 samples, Figure $1 E)$, respectively. DNA from paired PE cell sediment (PE-Sediments [terminated as PE-S], 30 samples) were fragmented to a size range from $150 \mathrm{bp}$ to $250 \mathrm{bp}$ using Covaris M220 (Covaris, Woburn, MA). All DNA fragments were analyzed in a Bioanalyzer 2100 using the High Sensitivity DNA Kit (Agilent Technologies, Santa Clara, CA). The sequencing library was constructed using the NEBNext Ultra II DNA Library Prep Kit for Illumina (New England BioLabs, Beverly, MA) using the standard protocol. Pre-capture libraries were established after end-repairing, A-tailing, adaptor ligation and PCR with indexed primers, all following the manufacturer's recommendations for probe hybridization and targeted capture with the AmoyDx pan-cancer 448-gene panel (Table S1) and lung cancer 10-gene panel (Table S2). Captured libraries were purified and amplified to obtain the post-capture library. Final sequencing of the library was obtained through quality assessment and using quantitative real-time polymerase chain reaction (PCR).
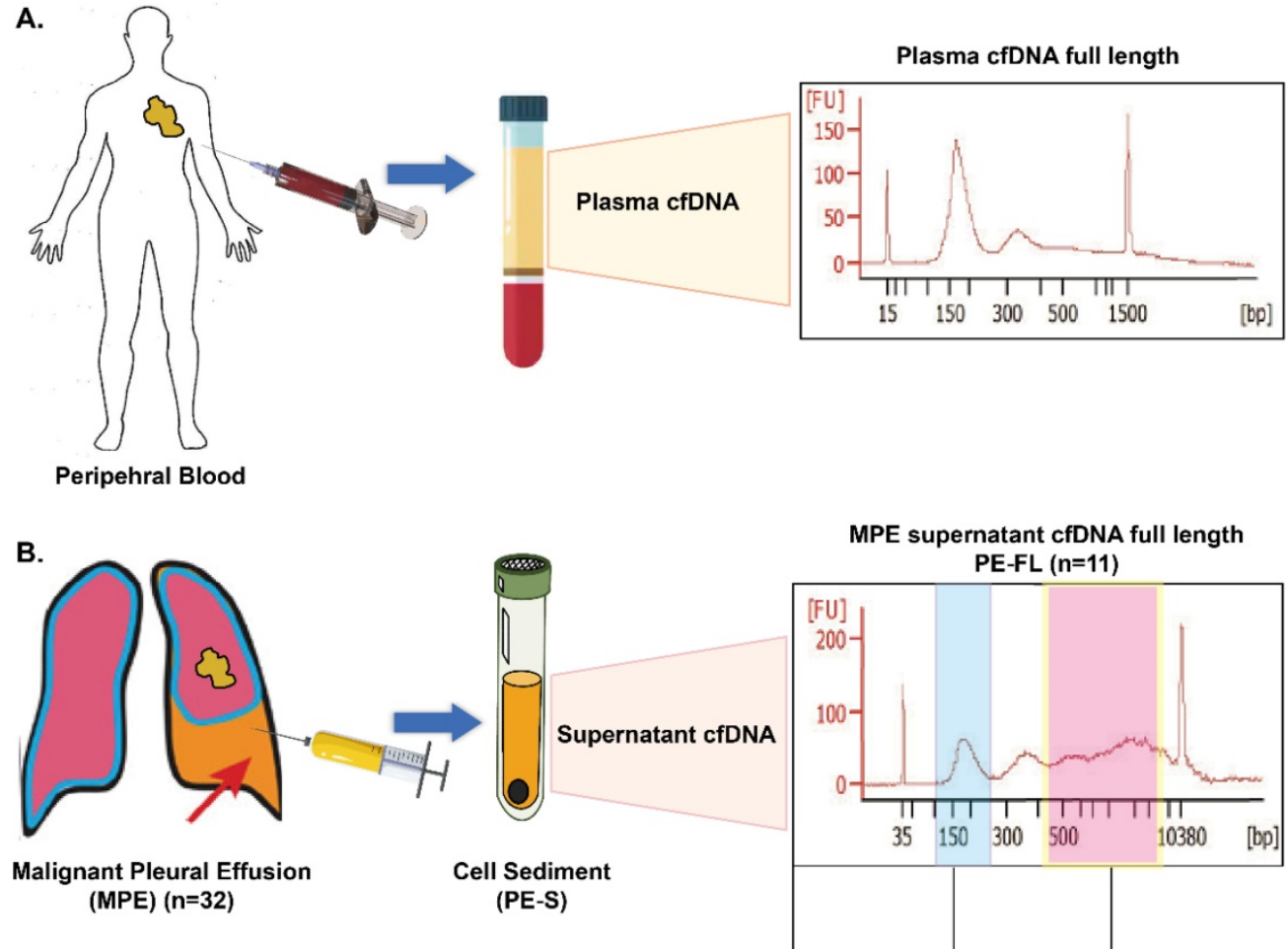

MPE supernatant cfDNA full length

B.

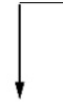

C. MPE Supernatant Full length DNA fragmented to $150-250 \mathrm{bp}$

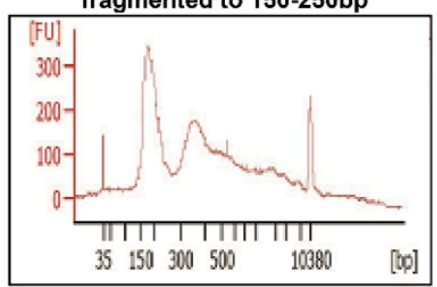

PE-F $(n=30)$
D.

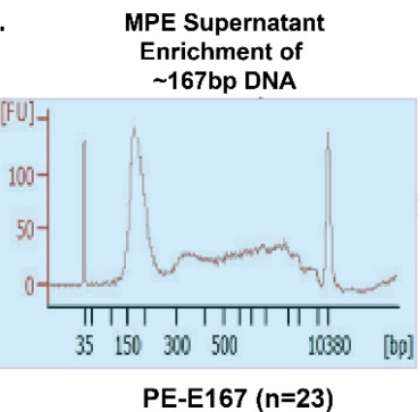

E.
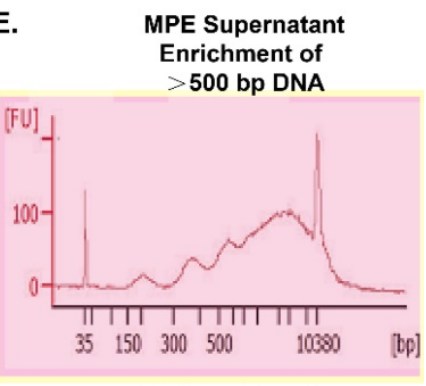

PE-E500 (n=6)

Figure 1. Distinct cfDNA profile in Malignant pleural effusion. A. Cell-free DNA (cfDNA) distribution in plasma as reference. B. Distribution of full length cfDNA in malignant pleural effusion (PE-FL). C. cfDNA in malignant pleural effusion fragmented to a size range from 150 bp to 250 bp (PE-F). D. Enriched 167 bp cfDNA fragment from malignant pleural effusion. E. Enriched cfDNA fragment larger than 500 bp. 


\section{Sequencing and data processing}

All normalized libraries were pooled, and DNA was sequenced in an Illumina NovaSeq 6000 platform with $2 \times 150-b p$ pair-end reads. Sequencing data were first cleaned to remove sequencing adaptors and low-quality reads (quality < 15) or poly-N with Trimmomatic and mapped to human reference genome, version 19 (hg19) using the BurrowsWheeler Aligner. PCR duplicates were marked and removed using Mark Duplicates from the Genome Analysis Toolkit (GATK). Base Quality Score Recalibration was performed using GATK's BaseRecalibrator and ApplyBQSR. After correction, a bam file was acquired. The InDels and single-nucleotide polymorphisms were compared by Mutect2 and FilterMutectCalls of GATK to obtain the final vcf file.

For analyzing hotspot mutations, the variant allele frequency (VAF) cutoff was $0.1 \%$ for both 10-gene and 448-gene panel [10]. ANOVA was used to annotate the vcf file into a MAF file to filter out common mutations and germline mutations. The MAF file was used for subsequent data analysis.

Referred to the algorithm of plasma bTMB in 2018 [13], the TMB of PE samples was determined by identifying all SNVs at an VAF either $\geq 0.5 \%$ or $0.1 \%$ as proposed by Tong et,al [10] across the coding region of 448 genes $(\sim 1.4 \mathrm{Mb})$ and filtering out germline mutations by paired blood samples. In addition, the 1000 Genomes and gnomAD database were compared to remove germline mutations with a frequency greater than $1 \%$ in healthy individuals.

\section{Statistical analyses}

The paired Student's $t$ test was applied for continuous variables in box plot and violin plot. All statistical analyses were performed using SPSS 23.0 (IBM, Armonk, NY) and R (version 4.0.1; The R Foundation, Vienna, Austria; http://www.R-project. org).

\section{Results}

\section{Patient baseline characteristics}

This study enrolled 32 patients with stage IV lung adenocarcinoma who had MPE at diagnosis or during disease progression (Table 1). The median age at enrollment was 58 years (range 43-77). 17 patients $(60.7 \%)$ were smokers. Smoking history of four patients was not available. More than half of the patients were male $(21,65.6 \%)$, and $10(31.2 \%)$ had EGFR mutations. 15 patients at clinical stage IVA (M1a stage, 25\%; M1b stage, 21.9\%) and 17 at clinical stage IVB (M1c stage, 53.1\%). All patients were pathologically confirmed adenocarcinoma.
Table 1. Clinical characteristics of patients with advanced lung cancer

\begin{tabular}{lc}
\hline Clinical Character $(\mathrm{n}=32)$ & Number $(\%)$ \\
\hline Gender (\%) & $11(34.4)$ \\
Female & $21(65.6)$ \\
Male & \\
Age (\%) & $15(46.9)$ \\
$<58$ & $17(53.1)$ \\
$\geq 58$ & \\
Smoking history (\%) & $13(46.4)$ \\
Current & $4(14.3)$ \\
Former & $11(39.3)$ \\
Never & \\
Clinical stage (\%) & $15(46.9)$ \\
IVA & $17(53.1)$ \\
IVB & \\
T stage (\%) & $8(25.0)$ \\
T1 & $13(40.6)$ \\
T2 & $4(12.5)$ \\
T3 & $7(21.9)$ \\
T4 & \\
N stage (\%) & $1(3.1)$ \\
N1 & $15(46.9)$ \\
N2 & $16(50.0)$ \\
N3 & \\
M stage (\%) & $9(28.1)$ \\
M1a & $6(18.8)$ \\
M1b & $17(53.1)$ \\
M1c &
\end{tabular}

\section{Cell-free DNA in malignant pleural effusion displayed distinct profile}

Among 32 samples, cell-free DNA were successfully extracted from 30 samples with accepted quality. DNA fragments were analyzed via Bioanalyzer 2100 yielding a distinct cfDNA distribution in PE supernatant, especially containing longer DNA fragments which had not been observed in plasma cfDNA (Figure 1A, 1B). To verify whether the long DNA fragments in the MPE supernatant will affect genetic test results including driver mutations as well as tumor mutation burden, full-length cfDNA from PE supernatant (PE-F) were fragmented to sizes ranging from $150 \mathrm{bp}$ to $250 \mathrm{bp}$ (Figure 1C) assuming including entire genetic information in MPE, followed by being tested via a 10-gene and a 448-gene panel respectively. The results were compared with those from either PE-E167 or PE-FL as well as PE-E500 (Figure 1B, 1D, and 1E). Meanwhile, genetic profiling was performed simultaneously for whole set of $\mathrm{PE}$ samples (PE-F, PE-E167, PE-E500 and PE-FL) from 4 patients.

\section{Fragmented full-length cfDNA (PE-F) displayed comparable hot-spot driver mutations with PE-FL or PE-E167}

No significant difference of hot spot driver mutations was found between two comparison categories (Figure 2A and 2B for PE-F vs PE-E167, Figure $2 \mathrm{C}$ and $2 \mathrm{D}$ for PE-F vs PE-FL) no matter via 
10-gene or 448-gene panels. Further, variant allele frequency (VAF) of mutations in PE-F has no difference compared with PE-E167 or PE-FL (Figure 2E, 2H). More hotspot variations with statistical significance were detected in both of PE-F, PE-E167 or PE-FL than paired PE-S (Figure 2E, 2H, p $<0.05$, respectively).

\section{Fragmented full-length cfDNA (PE-F) displayed unusual TMB distribution}

Validated Plasma bTMB algorithm [13] was applied to calculate pleural effusion-derived TMB for cfDNA in two groups, group A consisting of paired PE-F, and PE-E167 ( $n=23 ;$ P1-P7 \& P16, P18-P32), group $B$ consisting of paired PE-F and PE-FL $(n=11$; P4-P15). TMB calculated from PE-F were significantly higher than that calculated from either PE-E167 (Group A, median TMB for PE-F vs PE-E167, 17.8 mut $/ \mathrm{Mb}$ vs 7.8 mut/Mb, $\mathrm{p}<0.05$ ) (Figure $3 \mathrm{~A}$ ) or PE-FL (Group B median TMB for PE-F vs PE-FL, 35.1
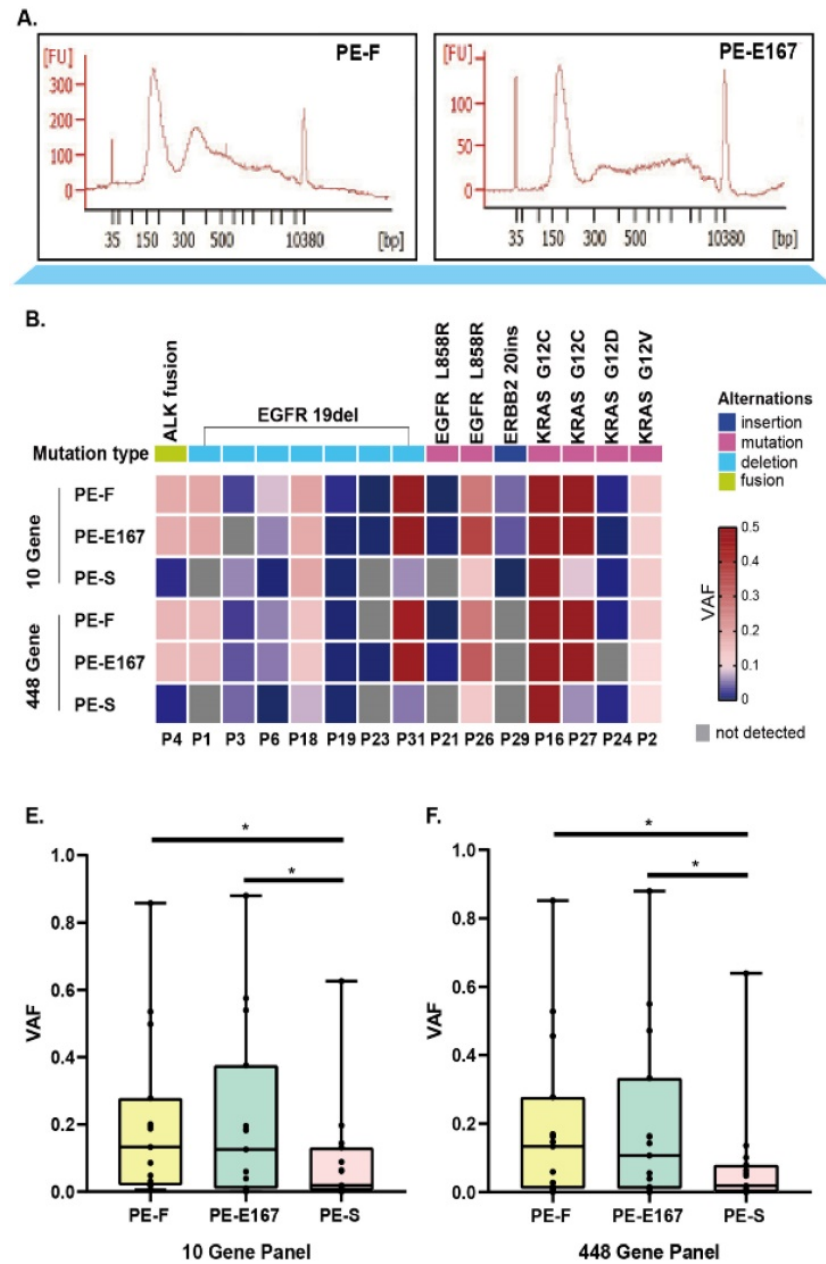

mut/ $\mathrm{Mb}$ vs 7.8 mut/Mb, $\mathrm{p}<0.05$ ) (Figure 3B). Medium TMB from entire PE-F cfDNA samples in this study $(20$ mut/ $\mathrm{Mb})$ was much higher than TMB reported by Tong et al. (6.4 mut/Mb) as well as medium TMB value in NSCLC. TMB calculated from variants with $\mathrm{VAF} \geq 0.1 \%$ also displayed significantly higher value in PE-F samples (Figure S1). These data suggested that the genetic information from long-fragment cfDNA in PE supernatant may interfere with TMB results.

\section{Distinct cfDNA profile, especially long-fragment cfDNA contained interfering variants affecting the accuracy of genetic testing}

Thus, it was hypothesized that long fragments of cfDNA contained in PE-F might influence the results of genetic analysis. Variations in driver genes with VAF $\geq 0.5 \%$ was compared between PE-E167 and PE-F by NGS 10-gene panel. Paired PE-E500 samples were
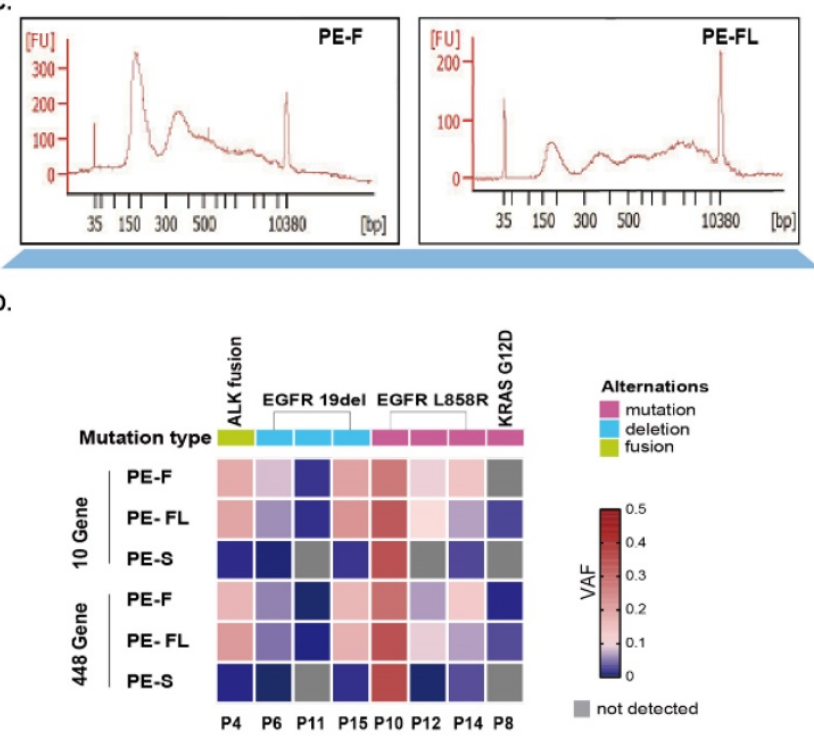

G.

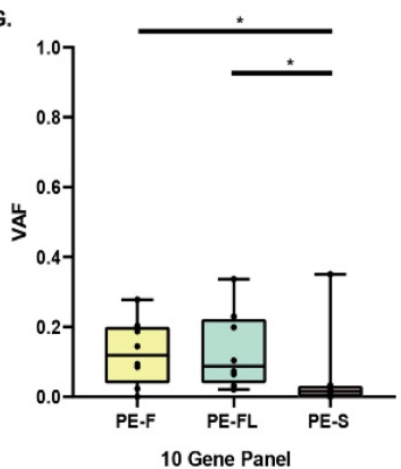

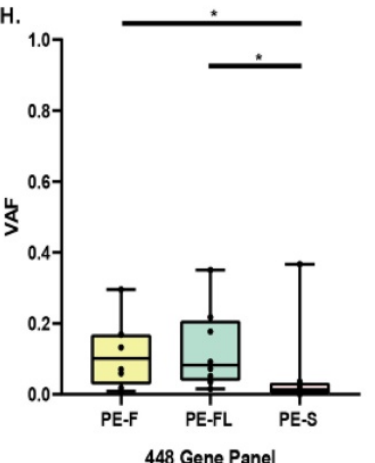

Figure 2. Comparing hotspot variants and tumor mutation burden among PE-E167, PE-FL, PE-F and PE-S. A. Comparing hotspot result between PE-F and PE-E167 samples. B. Hotspot variants detection rate of driver genes in PE-F, PF-E167 and PE-S using an NGS 10-gene and 448-gene panel. Variation types and VAF are indicated by different colors. C. Comparing hotspot result between PE-F and PE-FL samples. D. Hotspot variants detection rate of driver genes in PE-F, PF-FL and PE-S using an NGS 10-gene and 448-gene panel. Variation types and VAF are indicated by different colors. E. VAF of PE-F and PE-E167 were significantly higher than that of PE-S in NGS 10 -gen panel, $*_{p}<0.05$. F. VAF of PE-F and PE-E167 were significantly higher than that of PE-S in NGS 448-gen panel, * $P<0.05$. G. VAF of PE-F and PE-FL were significantly higher than that of PE-S in NGS 10-gene panel, * $p<0.05$. H. VAF of PE-F and PE-FL were significantly higher than that of PE-S in NGS 448 -gene panel, * $P<0.05$. 
considered as the source of long-fragment cfDNA. Hotspot variations in drivers as marked by green dots above the X-axis were mainly observed in PE-167 group; While Unknown variations, marked with brown dots below the $\mathrm{X}$-axis, were enriched in PE-F and PE-E500 samples (Figure 4A). Referring to the COSMIC database (Catalogue of Somatic Mutation in Cancer, https://cancer.sanger.ac.uk/cosmic), more than half of the variants in 10 NSCLC driver genes had not been reported (data not shown). Moreover, majority of the variants detected by comprehensive genomic profiling in PE-F and PE-E500 were not reported by COSMIC no matter with $\mathrm{VAF} \geq 0.5 \%$ or $0.1 \%$ (Figure 4B, 4D, Figure S2A, S2D, and S2E). Especially, allele frequency of these COSMIC unarchived variants was majorly less than $1 \%$ in 30 PE-F samples or PE-E500 from 4 paired samples (Figure S2B, S2C).

Therefore, these unknown variations, majorly $\mathrm{VAF}<1 \%$, suspected to be interference introduced by long-fragment cfDNA, will affect accuracy of evaluating $\mathrm{TMB}$ if $\mathrm{VAF} \geq 0.5 \%$ or $0.1 \%$ was set as cut-off value.

\section{Similar genetic profile was observed in paired PE-FL and PE-E167 cfDNA samples}

PE-FL and PE-E167 cfDNA samples from two comparison groups yielded comparable TMB value. In addition, results from PE-FL and PE-E167 showed similar efficacy guiding tyrosine kinase inhibitor treatment as compared to result from clinical routine test (Figure S3).

To clarify the concordance of genetic profile between these two cfDNA categories, paired PE-FL and PE-E167 were collected. Numbers of mutations were similar under three VAF cut-offs $(0.1 \%, 0.5 \%$ and 1\%) (Figure 5A). In parallel, PE-FL and PE-E167 displayed almost same profile of genetic mutations (Figure 5B). Therefore, it is recommended to use PE-FL to prepare sequencing library without the need
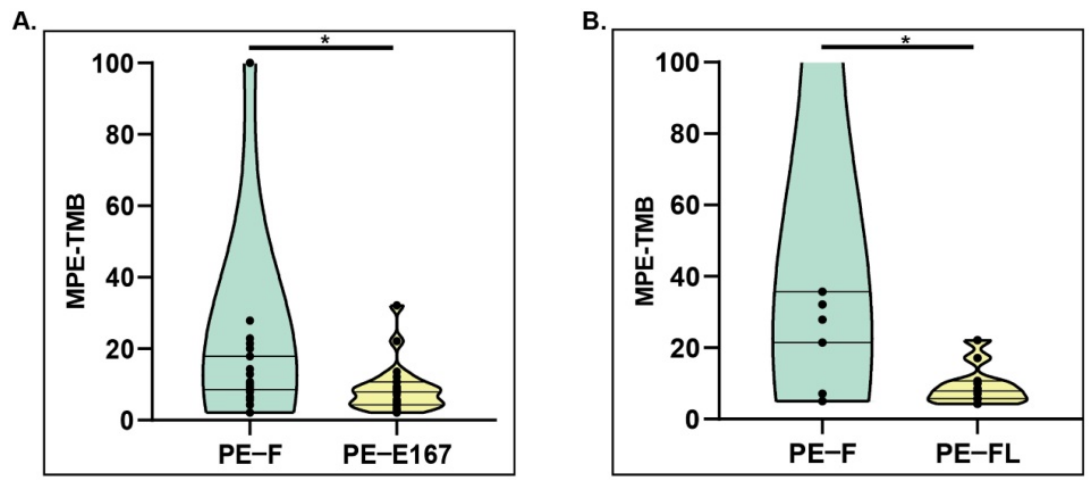

Figure 3. Comparing TMB distribution among various sample types. A. PE-TMB was calculated using the plasma $b T M B$ algorithm. The TMB calculated from PE-F were significantly higher than that calculated from $\mathrm{PE}-\mathrm{E} 167,{ }^{*} \mathrm{P}<0.05$. B. The TMB calculated from PE-F were significantly higher than that calculated from PE-FL, $*_{p}<0.05$. for fragmentation of full-length cfDNA in clinical practice.

\section{Discussion}

This study demonstrated at first time the distinct profile of cfDNA in malignant pleural effusion. This unique character, especially long cfDNA fragments in supernatant of pleural effusion showed potential impact on genetic analysis, especially TMB evaluation via introducing large number of interfering, non-cancer specific variants. Both use of total PE-cfDNA without fragmentation step and enriched PE-cfDNA at $\sim 167$ bp for NGS library preparation can avoid interference from long cfDNA fragments giving reliable and comparable results.

Pleural Effusion, as obtained by pleural drainage in clinics, besides liquid-based cytology (LBC) detecting cancer cells in patients with advanced cancer $[14,15]$ it has been previously proved to be suitable material for histological and genetic analysis [16-18]. However, several obstacles including various numbers of tumor cells as well as leukocytes in PE sample, especially PE sediment, limited the sensitivity for detection of somatic variations. Thus, cfDNA in the supernatant of pleural effusion samples may be surrogate for molecular profiling. In this study, consistent with previous studies [5, 10, 19], PE supernatant cfDNA is a valuable surrogate material for detecting hotspot variations and TMB. Indeed, PE supernatant cfDNA was significantly superior to PE sediments (PE-S) in mutation detection. Thus, compared with PE sediment, PE supernatant cfDNA may be a better source of DNA without influence from other cell types like leukocyte, allowing genetic analysis available for patients with advanced lung cancer.

Genetic analysis of circulating cfDNA has been widely used in molecular diagnostics. DNA fragments within a specific range of size need to be isolated. Distribution of DNA fragments in PE supernatant has not been examined. Damaged tumor cells together with apoptotic or pyroptotic leukocytes may release large amounts of cfDNA in the pleural space, making pleural fluid a possible rich source of cfDNA. As Tong et al. reported, the amount of DNA was significantly higher in PE than in plasma. Therefore, analyzing and selecting cfDNA fragment in PE supernatant may help to improve the accuracy of genetic analysis. Our results have shown that longer DNA fragments in the PE supernatant cfDNA compared 
with plasma. This finding has not been reported so far. In lung cancer, the detection of hotspot mutations in driver genes such as EGFR was used as guidance for targeted therapy [20]. On the other hand, the TMB value can be used to evaluate the efficacy of immunotherapy [21]. Therefore, hotspot mutations and $\mathrm{TMB}$ were used as evaluative indicators to determine the influence from these long cfDNA fragments. No difference of driver gene mutations was observed between PE-F and PE-E167 or PE-FL by NGS lung cancer 10-gene panel or 448-cancer related gene panel. PE-F and PE-E500 detected more cancer-unrelated variations not archived in COSMIC, majorly with VAF ranging from $0.1 \%-1 \%$, suggesting long-fragment cfDNA in PE supernatant may influence comprehensive genomic profiling. PE-F samples gave significantly higher TMB values which proved this concept.

To avoid interference from long fragments in PE-cfDNA, direct use of total PE-cfDNA without fragmentation step is recommended to construct the NGS library like PE-FL in this study as long fragments cannot be successfully captured and eventually excluded from the library. Another way is to enrich cfDNA fragments at $\sim 167 \mathrm{bp}$ like PE-E167. Both of these two approaches can give trustable hotspot mutation result as well as reliable TMB evaluation. Enriching $\sim 167 \mathrm{bp}$ cfDNA fragment may cost extra time, thus, directly using total PE-cfDNA without fragmentation step to prepare sequencing library is preferred in clinical practice.

This study has several limitations. First, the limited sample size might produce statistical bias. A larger study cohort for technical and clinical validation is warranted. Second, no paired tissue samples were available as "gold standard" for PE supernatant cfDNA tested result. Third, what is the potential biological mechanism for the generation of low-VAF non-tumor mutations has not been investigated in this study.
A.
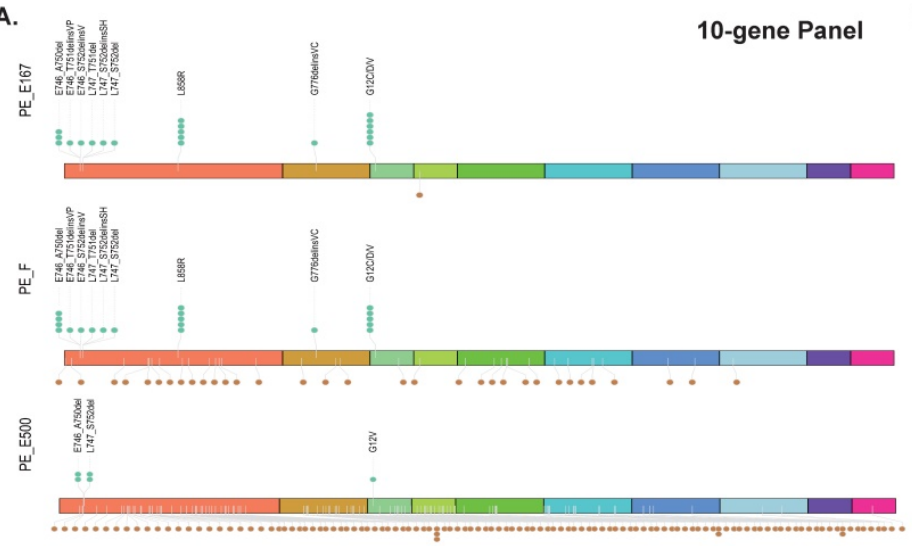

$\square$ EGFR $\square$ ERBB2 $\square$ KRAS $\square$ MET $\square$ ALK $\square$ NRAS $\square$ PIK3CA $\square$ RET $\square$ BRAF $\|$ ROS1

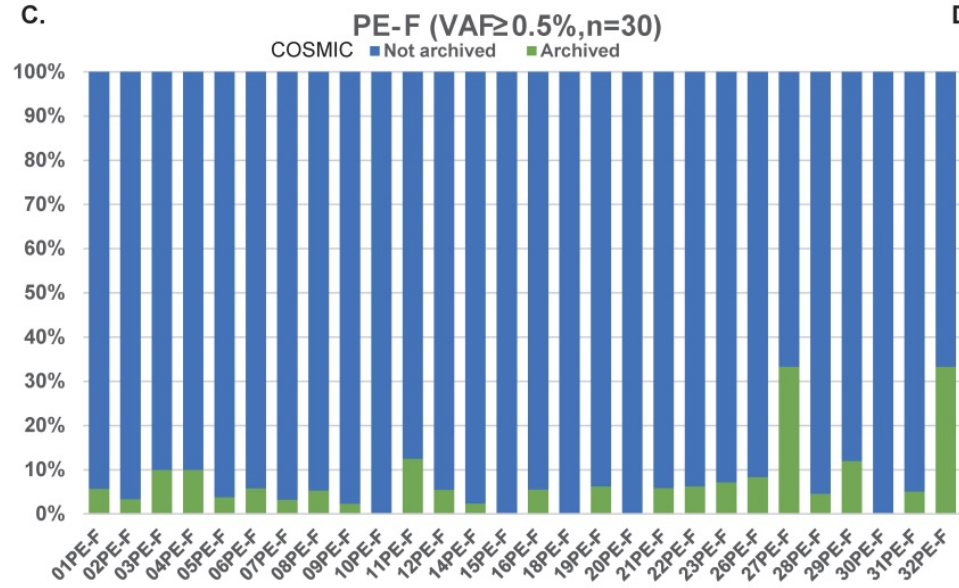

B.
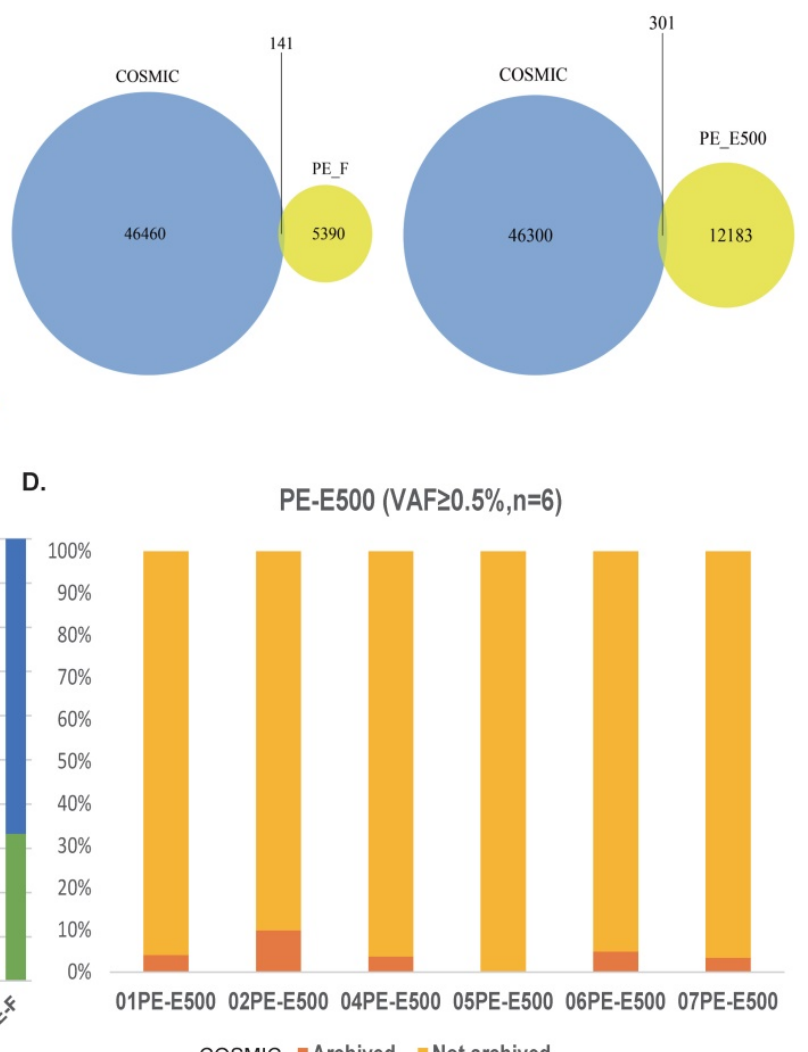

Figure 4. Distinct cfDNA profile, especially long-fragment cfDNA in malignant pleural effusion contains COSMIC unreported variants. A. An NGS 10-gene panel was used to compare the differences in the detected mutation sites between PE-E167, PE-F and PE-E500. 10 lung cancer-related driver genes were examined in PE-E167, PE-F and PE-E500. Hotspot variants are labeled green above each X-axis, unknown variations are labeled brown below each X-axis. B. Left panel: Venn graph displayed major variants with $\mathrm{VAF} \geq 0.5 \%$ in PE-F samples were not reported and archived by COSMIC database. Right panel: Venn graph displayed major variants with $\mathrm{VAF} \geq 0.5 \%$ in $\mathrm{PE}-\mathrm{E} 500$ samples were not reported and archived by COSMIC database. C. Bar graph displayed distribution of COSMIC unarchived variants with VAF $\geq 0.5 \%$ in each PE-F sample. D. Bar graph displayed distribution of COSMIC unarchived variants with $\mathrm{VAF} \geq 0.5 \%$ in six PE-E500 samples. 
A.
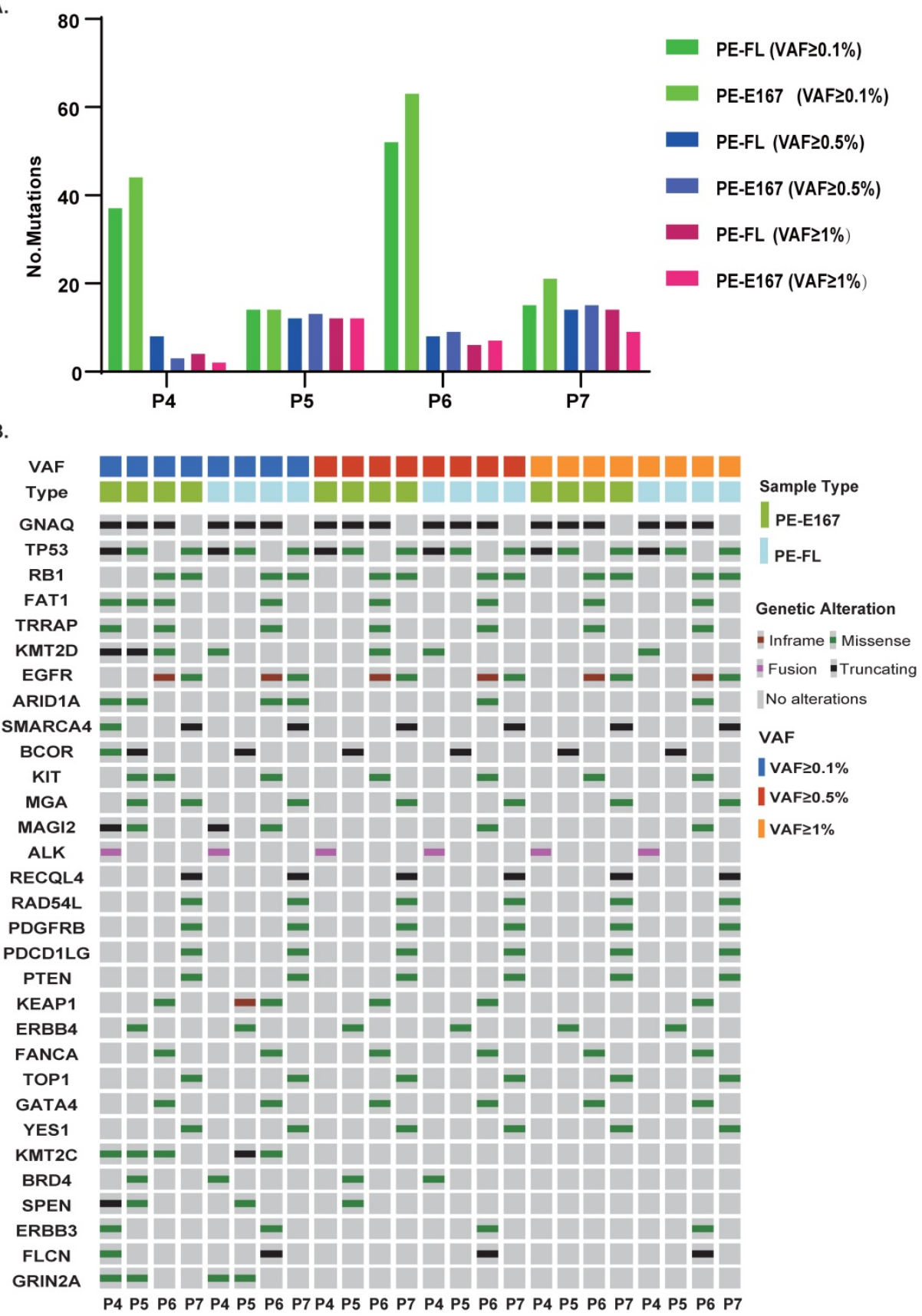

Figure 5. Paired PE-E167 and PE-FL showed comparable genetic profile. A. Bar plot shows distribution of detected mutation number under various VAF cut-offs in paired PE-E167 and PE-FL from four patients. B. Mutation profile under various VAF cut-offs in paired PE-E167 and PE-FL from four patients.

In conclusion, our data shown that long cfDNA fragments are more contained in PE supernatants than that in plasma cfDNA. Although PE-F can be used for detecting hotspot mutations, long fragments will affect the accuracy of test results, especially TMB by introduction of cancer-unrelated variants; especially with low allele frequency $(0.1 \%-1 \%)$. Direct use of total PE-cfDNA without fragmentation step to construct sequencing library, instead of using fragmented full-length PE-cfDNA is recommended for clinical practice.

\section{Abbreviations}

bTMB: blood tumor mutational burden; cfDNA: cell free DNA; EGFR: epidermal growth factor receptor; GATK: Genome Analysis Toolkit; gDNA: genomic DNA; LBC: liquid-based cytology; MPE: malignant pleural effusion; NGS: next-generation sequencing; NSCLC: non-small cell lung cancer; PCR: polymerase chain reaction; PE: pleural effusion; PE-E167: PE cfDNA enriched to 167 bp; PE-E500: PE cfDNA fragment larger than 500 bp; PE-F: full-length cfDNA fragmented to 150-250 bp; PE-FL: full-length 
cfDNA from PE supernatant without fragmentation step during NGS library preparation; TMB: tumor mutational burden; VAF: variant allele frequency.

\section{Supplementary Material}

Supplementary figures and tables.

http://www.medsci.org/v18p1510s1.pdf

\section{Acknowledgements}

This work was supported by the National Key R\&D Program of China (2016YFC1303300), the National Natural Science Foundation of China (81672272), Shanghai Municipal Science \& Technology Commission Research Project (17431906103), Shanghai Chest Hospital Project of Collaborative Innovation (YJXT20190105), Clinical Research Plan of SHDC (16CR3005A), and Linghang Cancer Research Project of CSCO.

We thank Ziwei Fan, Zhan Huang (Amoy Diagnostics Co., Ltd., Xiamen, China) for data extraction, statistical analysis and processing.

\section{Competing Interests}

The authors have declared that no competing interest exists.

\section{References}

1. Mazieres J, Drilon A, Lusque A, Mhanna L, Cortot AB, Mezquita L, et al. Immune checkpoint inhibitors for patients with advanced lung cancer and oncogenic driver alterations: results from the IMMUNOTARGET registry. Ann Oncol. 2019; 30: 1321-8.

2. Yoda S, Dagogo-Jack I, Hata AN. Targeting oncogenic drivers in lung cancer: Recent progress, current challenges and future opportunities. Pharmacol Ther. 2019; 193: 20-30

3. Arbour KC, Riely GJ. Systemic Therapy for Locally Advanced and Metastatic Non-Small Cell Lung Cancer: A Review. JAMA. 2019; 322: 764-74.

4. Wang Z, Zhang L, Li L, Li X, Xu Y, Wang M, et al. Sputum Cell-Free DNA: Valued Surrogate Sample for Detection of EGFR Mutation in Patients with Advanced Lung Adenocarcinoma. The Journal of molecular diagnostics : JMD. 2020; 22: 934-42.

5. Villatoro S, Mayo-de-Las-Casas C, Jordana-Ariza N, Viteri-Ramirez S, Garzon-Ibanez M, Moya-Horno I, et al. Prospective detection of mutations in cerebrospinal fluid, pleural effusion, and ascites of advanced cancer patients to guide treatment decisions. Molecular oncology. 2019; 13: 2633-45.

6. Merker JD, Oxnard GR, Compton C, Diehn M, Hurley P, Lazar AJ, et al. Circulating Tumor DNA Analysis in Patients With Cancer: American Society of Clinical Oncology and College of American Pathologists Joint Review. J Clin Oncol. 2018; 36: 1631-41.

7. Psallidas I, Kalomenidis I, Porcel JM, Robinson BW, Stathopoulos GT. Malignant pleural effusion: from bench to bedside. Eur Respir Rev. 2016; 25: 189-98.

8. Kawahara A, Fukumitsu C, Azuma K, Taira T, Abe H, Takase Y, et al. A Combined test using both cell sediment and supernatant cell-free DNA in pleural effusion shows increased sensitivity in detecting activating EGFR mutation in lung cancer patients. Cytopathology. 2018; 29: 150-5.

9. Ozcakar B, Martinez CH, Morice RC, Eapen GA, Ost D, Sarkiss MG, et al. Does pleural fluid appearance really matter? The relationship between fluid appearance and cytology, cell counts, and chemical laboratory measurements in pleural effusions of patients with cancer. J Cardiothorac Surg. 2010; 5: 63

10. Tong L, Ding N, Tong X, Li J, Zhang $Y$, Wang $X$, et al. Tumor-derived DNA from pleural effusion supernatant as a promising alternative to tumor tissue in genomic profiling of advanced lung cancer. Theranostics. 2019; 9: 5532-41.

11. Jiang P, Lo YMD. The Long and Short of Circulating Cell-Free DNA and the Ins and Outs of Molecular Diagnostics. Trends Genet. 2016; 32: 360-71.

12. Jahr S, Hentze H, Englisch S, Hardt D, Fackelmayer FO, Hesch RD, et al. DNA fragments in the blood plasma of cancer patients: quantitations and evidence for their origin from apoptotic and necrotic cells. Cancer Res. 2001; 61: 1659-65.

13. Gandara DR, Paul SM, Kowanetz M, Schleifman E, Zou W, Li Y, et al. Blood-based tumor mutational burden as a predictor of clinical benefit in non-small-cell lung cancer patients treated with atezolizumab. Nat Med. 2018; 24: $1441-8$

14. Lee YM, Hwang JY, Son SM, Choi SY, Lee HC, Kim EJ, et al. Comparison of diagnostic accuracy between CellprepPlus(R) and ThinPrep(R) liquid-based preparations in effusion cytology. Diagn Cytopathol. 2014; 42: 384-90.

15. Zhao FH, Hu SY, Bian JJ, Liu B, Peck RB, Bao YP, et al. Comparison of ThinPrep and SurePath liquid-based cytology and subsequent human papillomavirus DNA testing in China. Cancer Cytopathol. 2011; 119: 387-94.

16. Wu W, Cao Z, Zhang W, Zhang L, Hou L, Wu C. Comparison of the SuperARMS and ARMS for detecting EGFR mutations in liquid-based cytology specimens from NSCLC patients. Diagn Pathol. 2020; 15: 9.

17. Roy-Chowdhuri S, Aisner DL, Allen TC, Beasley MB, Borczuk A, Cagle PT, et al. Biomarker Testing in Lung Carcinoma Cytology Specimens: A Perspective From Members of the Pulmonary Pathology Society. Arch Pathol Lab Med. 2016; 140: 1267-72.

18. Ellison G, Zhu G, Moulis A, Dearden S, Speake G, McCormack R. EGFR mutation testing in lung cancer: a review of available methods and their use for analysis of tumour tissue and cytology samples. J Clin Pathol. 2013; 66: $79-89$

19. Lee JS, Hur JY, Kim IA, Kim HJ, Choi CM, Lee JC, et al. Liquid biopsy using the supernatant of a pleural effusion for EGFR genotyping in pulmonary adenocarcinoma patients: a comparison between cell-free DNA and extracellular vesicle-derived DNA. BMC Cancer. 2018; 18: 1236.

20. Piper-Vallillo AJ, Sequist LV, Piotrowska Z. Emerging Treatment Paradigms for EGFR-Mutant Lung Cancers Progressing on Osimertinib: A Review. Journal of clinical oncology: official journal of the American Society of Clinical Oncology. 2020: JCO1903123.

21. Sholl LM, Hirsch FR, Hwang D, Botling J, Lopez-Rios F, Bubendorf L, et al. Tumor Mutation Burden: Promises and Challenges A Perspective from the IASLC Pathology Committee. Journal of thoracic oncology : official publication of the International Association for the Study of Lung Cancer. 2020. 